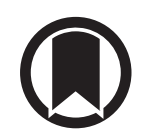

CrossMark

\title{
Incident and prevalent cohorts with pulmonary arterial hypertension: insight from SERAPHIN
}

\author{
Gérald Simonneau ${ }^{1,2,3}$, Richard N. Channick ${ }^{4}$, Marion Delcroix ${ }^{5}$, \\ Nazzareno Galié6, Hossein-Ardeschir Ghofrani ${ }^{7,8,9}$, Pavel Jansa ${ }^{10}$, \\ Franck-Olivier Le Brun ${ }^{11}$, Sanjay Mehta ${ }^{12}$, Loic Perchenet ${ }^{11}$, Tomás Pulido ${ }^{13}$, \\ B.K.S. Sastry ${ }^{14}$, Olivier Sitbon ${ }^{1,2,3}$, Rogério Souza ${ }^{15}$, Adam Torbicki ${ }^{16}$ and \\ Lewis J. Rubin ${ }^{17}$
}

\begin{abstract}
Affiliations: ${ }^{1}$ Assistance Publique-Hôpitaux de Paris, Service de Pneumologie, Hôpital Bicêtre, Le KremlinBicêtre, France. ${ }^{2}$ Université Paris-Sud, Laboratoire d'Excellence en Recherche sur le Médicament et Innovation Thérapeutique, Le Kremlin-Bicêtre, France. ${ }^{3}$ INSERM U-999, Centre chirurgical Marie Lannelongue, Le Plessis Robinson, France. ${ }^{4}$ Pulmonary and Critical Care, Massachusetts General Hospital, Boston, MA, USA. ${ }^{5}$ Department of Pneumology, Gasthuisberg University Hospital, Leuven, Belgium. ${ }^{6}$ Department of Experimental, Diagnostic and Specialty Medicine (DIMES), Bologna University Hospital, Bologna, Italy. ${ }^{7}$ University of Giessen and Marburg Lung Center, Giessen, Germany. ${ }^{8}$ Member of the German Center of Lung Research (DZL), Giessen, Germany. ${ }^{9}$ Department of Medicine, Imperial College London, London, UK. ${ }^{10} \mathrm{Clinical}$ Department of Cardiology and Angiology, 1st Faculty of Medicine, 2nd Medical Department, Charles University, Prague, Czech Republic. ${ }^{11}$ Actelion Pharmaceuticals Ltd, Allschwil, Switzerland. ${ }^{12}$ Division of Respirology, Department of Medicine, London Health Sciences Centre - Victoria Hospital, Western University, London, ON, Canada. ${ }^{13}$ Cardiopulmonary Department, Ignacio Chávez National Heart Institute, Mexico City, Mexico. ${ }^{14}$ Department of Cardiology, CARE Hospitals, Hyderabad, India. ${ }^{15}$ Pulmonary Department, Heart Institute, University of São Paulo Medical School, São Paulo, Brazil. ${ }^{16}$ Department of Pulmonary Circulation and Thromboembolic Diseases, Center of Postgraduate Medical Education, ECZ-Otwock, Otwock, Poland.

${ }^{17}$ Division of Pulmonary and Critical Care Medicine, University of California, San Diego Medical School, San Diego, CA, USA.
\end{abstract}

Correspondence: Gérald Simonneau, Assistance Publique-Hôpitaux de Paris, Service de Pneumologie, Hôpital Bicêtre; Université Paris-Sud, Laboratoire d'Excellence en Recherche sur le Médicament et Innovation Thérapeutique, and INSERM U-999, 78 Rue du Général Leclerc, 94270 Le Kremlin-Bicêtre, France.

E-mail: gerald.simonneaudabct.aphp.fr

ABSTRACT In SERAPHIN, a long-term, randomised, controlled trial (NCT00660179) in pulmonary arterial hypertension (PAH), macitentan significantly reduced the risk of morbidity/mortality and PAHrelated death/hospitalisation. We evaluated disease progression and the effect of macitentan in treatmentnaïve incident and prevalent cohorts.

Patients allocated to placebo, or macitentan $3 \mathrm{mg}$ or $10 \mathrm{mg}$ were classified by time from diagnosis to enrolment as incident ( $\leqslant 6$ months; $n=110)$ or prevalent ( $>6$ months; $n=157)$. The risk of morbidity/ mortality and PAH-related death/hospitalisation was determined using Cox regression.

The risk of morbidity/mortality (Kaplan-Meier estimates at month 12: 54.4\% versus 26.7\%; $\mathrm{p}=0.006$ ) and PAH-related death/hospitalisation (Kaplan-Meier estimates at month 12: 47.3\% versus 19.9\%; $\mathrm{p}=0.006$ ) were significantly higher for incident versus prevalent patients receiving placebo, respectively. There was no significant difference in the risk of all-cause death between incident and prevalent cohorts ( $\mathrm{p}=0.587$ ). Macitentan $10 \mathrm{mg}$ significantly reduced the risk of morbidity/mortality and PAH-related death/ hospitalisation versus placebo in incident and prevalent cohorts.

Incident patients had a higher risk for PAH progression compared with prevalent patients but not a higher risk of death. Macitentan delayed disease progression in both incident and prevalent PAH patients.

@ERSpublications

In the SERAPHIN trial, incident PAH patients had a higher risk of disease progression than prevalent patients http://ow.ly/RvCei

Received: March 052015 | Accepted after revision: July 222015 | First published online: Oct 222015

Copyright $\odot$ ERS 2015. ERJ Open articles are open access and distributed under the terms of the Creative Commons Attribution Non-Commercial Licence 4.0. 


\section{Introduction}

Pulmonary arterial hypertension (PAH) is a severe, progressive disease leading to right heart failure. Advances in the management of $\mathrm{PAH}$, including the introduction of targeted therapies, have led to improved survival [1]. However, $\mathrm{PAH}$ remains progressive and fatal [2], highlighting the importance of further improvements in treatments and their effective implementation.

Patient and disease characteristics, such as male sex [2,3] and World Health Organization (WHO) functional class (FC) III or IV [2, 4], are predictors of poor prognosis. Data from national PAH registries in the US and Europe have also highlighted that newly diagnosed patients (termed the incident cohort) have a worse prognosis than those who are diagnosed before follow-up begins (termed the prevalent cohort) $[2,4]$. The US National Institutes of Health Registry of Primary Pulmonary Hypertension showed a median survival of 2.6 years for newly diagnosed patients compared with 3.2 years for previously diagnosed patients [4]. Differences in survival between incident and prevalent patients were observed in the French PAH registry, with 3 -year survival rates of $51 \%$ and $71 \%(p<0.0001)$ [2]. Furthermore, recent data from REVEAL (Registry to Evaluate Early and Long-term Pulmonary Arterial Hypertension Disease Management) in the USA estimate 3-year survival of $69.3 \%$ and $76.2 \%$ in incident and prevalent patients, which is comparable to the French cohort [5]. The improved survival observed in registries for prevalent patients may be influenced by survivor bias $[2,6,7]$.

Macitentan is a novel, oral endothelin receptor antagonist (ERA), characterised by sustained receptor binding $[8,9]$ and enhanced tissue penetration $[10,11]$, that is approved for the long-term treatment of $\mathrm{PAH}$ to delay disease progression [12]. In the large, randomised, controlled Study with an Endothelin Receptor Antagonist in Pulmonary Arterial Hypertension to Improve Clinical Outcome (SERAPHIN), macitentan $10 \mathrm{mg}$ reduced the risk of the composite end-point of morbidity and mortality by $45 \%$, and the risk of PAH-related death and hospitalisation by $50 \%$ [13].

The objective of the current analysis was to evaluate disease progression in incident and prevalent treatment-naïv PAH patients in the global SERAPHIN trial, and to study the effect of first-line treatment with macitentan on long-term outcomes in these patients.

\section{Methods}

Study design and patients

SERAPHIN was a global, double-blind, randomised, placebo-controlled, event-driven, phase III study designed to assess the long-term efficacy and safety of macitentan in patients with PAH (www.clinicaltrials.gov identifier number NCT00660179). The study has been described in detail elsewhere [13]. Patients were $\geqslant 12$ years of age with $\mathrm{PAH}$ diagnosed by right heart catheterisation, including idiopathic and heritable $\mathrm{PAH}$, or $\mathrm{PAH}$ associated with connective tissue disease, repaired simple congenital systemic-to-pulmonary shunts, HIV infection, or drugs and toxins. Patients were also required to be in WHO FC II-IV and have a 6-min walk distance $(6 \mathrm{MWD}) \geqslant 50 \mathrm{~m}$. Patients could be treatment naïve, or receiving a stable dose of phosphodiesterase type 5 inhibitors, oral or inhaled prostanoids, calcium channel blockers, or L-arginine for $\geqslant 3$ months. After screening, patients were randomised (1:1:1) to placebo, macitentan $3 \mathrm{mg}$ or macitentan $10 \mathrm{mg}$. Double-blind treatment was continued until patients experienced a primary end-point event or until 285 events had occurred (end of study (EOS)). An independent clinical event committee adjudicated, in a blinded fashion, all morbidity and mortality events that were reported up to the end of double-blind treatment, including whether death was due to PAH. Local institutional review boards or independent ethics committees approved the protocol. Written informed consent was obtained from all patients.

Exploratory post hoc analyses were conducted on the subgroup of treatment-naïve patients enrolled in SERAPHIN. Classification of patients into incident or prevalent cohorts was based on a cut-off for the time from PAH diagnosis to study enrolment. The incident cohort comprised treatment-naive patients, diagnosed $\leqslant 6$ months prior to enrolment. The prevalent cohort comprised treatment-naïve patients, diagnosed $>6$ months prior to enrolment. The 6-month threshold was used as it is the median of the range

This article has supplementary material available from erj.ersjournals.com

Clinical trial: This article is registered at www.clinicaltrials.gov with identifier number NCT00660179

Support statement: SERAPHIN was funded by Actelion Pharmaceuticals Ltd. Medical writing support was provided by Lisa Thomas of Elements Communications Ltd (Westerham, UK) (manuscript outline), Marion James and Jonathan Viney of Apothecom Scopemedical Ltd (London, UK) (manuscript), and Anoushka Thomas of nspm ltd (Meggen, Switzerland) (manuscript), funded by Actelion Pharmaceuticals Ltd. Funding information for this article has been deposited with FundRef.

Conflict of interest: Disclosures can be found alongside the online version of this article at erj.ersjournals.com 
used previously to define incident and prevalent PAH patients $[2,4,14-16]$ and is substantially lower than the 16-month cut-off used in the French PAH Registry [2]. In addition, the 6-month threshold ensured that a reasonable number of patients in each cohort were included. We examined the disease course in the incident and prevalent cohorts without intervention by analysing the outcome measures in placebo-treated patients. Patients receiving PAH therapy at baseline were excluded to remove the potential confounding effects of background PAH-specific therapies on the comparison between the incident and prevalent cohorts, and to evaluate the effect of first-line treatment with macitentan in these two cohorts. For the primary end-point, the robustness of the results in the treatment-naïve population was assessed by conducting the analysis on all incident and prevalent patients, regardless of PAH therapy at baseline.

\section{Outcome measures}

The primary end-point was the time from initiation of treatment with the study drug to the first morbidity (worsening of $\mathrm{PAH}$, initiation of intravenous/subcutaneous prostanoids, lung transplantation or atrial septostomy) or all-cause mortality event up to the end of double-blind treatment (EOT). Worsening of $\mathrm{PAH}$ was defined by the occurrence of all of the following: $\geqslant 15 \%$ decrease in $6 \mathrm{MWD}$ from baseline (confirmed by a second test performed on a different day and within 2 weeks), worsening of PAH symptoms and requirement for additional PAH treatment. A blinded independent committee adjudicated all primary end-point events. Secondary end-points included time from study drug initiation to the occurrence of death due to PAH or hospitalisation for PAH (PAH-related death or hospitalisation) up to EOT and time to all-cause mortality up to the EOS. Safety endpoints included adverse events and laboratory abnormalities.

\section{Statistical analysis}

The time-to-event end-points were analysed using the Kaplan-Meier method and comparisons between groups (placebo incident versus placebo prevalent or macitentan groups versus placebo within the incident and prevalent cohorts) were made using the log-rank test. For the comparisons between incident and prevalent placebo patients, baseline variables that differed between the two groups with $\mathrm{p}<0.20$ ( $\mathrm{t}$-test for continuous variables and Fisher test for categorical variables), together with PAH aetiology (which is prognostic of long-term outcome [17] and differed between the groups with a borderline p-value), were considered for inclusion into a multivariate Cox regression model as a supportive analysis. Both unadjusted and adjusted hazard ratios (HRs) with 95\% confidence intervals were calculated. In addition, for each end-point, interaction tests were performed in order to evaluate the consistency of the treatment effect for macitentan versus placebo between incident and prevalent cohorts.

\section{Results}

Patients and treatment exposure

Of the 742 patients enrolled in SERAPHIN, 267 were treatment-naive. Of these, 110 were considered incident and 157 were considered prevalent. Treatment-naïve PAH patients received either placebo (incident: $n=36$; prevalent: $n=59$ ), macitentan 3 mg (incident: $n=40$; prevalent: $n=45$ ) or macitentan $10 \mathrm{mg}$ (incident: $\mathrm{n}=34$; prevalent: $\mathrm{n}=53$ ). Statistical analysis was conducted to compare the baseline characteristics between incident and prevalent patients in each treatment group and overall. In the placebo incident and prevalent cohorts, differences at the $5 \%$ level were found in baseline geographical region and WHO FC (table 1). In the overall incident and prevalent cohorts, significant differences were found in baseline aetiology and geographical region (table 1). Both placebo cohorts were mainly comprised of European patients; the placebo incident cohort had a higher proportion of patients from Western Europe and a smaller proportion from Eastern Europe compared with the placebo prevalent cohort. A greater proportion of placebo incident patients were WHO FC III/IV compared with prevalent patients $(62.9 \%$ versus $37.3 \%$; table 1). Notwithstanding the time from diagnosis to enrolment, both cohorts were similar with regard to other baseline characteristics.

\section{Morbidity and mortality end-point}

The total number and type of morbidity and mortality end-point events experienced by patients in the incident and prevalent cohorts are shown by treatment group in table 2. Among the placebo-treated patients, a greater proportion of incident patients experienced a morbidity or mortality event compared with prevalent patients. The majority of these events were PAH worsening. Kaplan-Meier estimates of morbidity and mortality at month 12 were $54.4 \%$ for the incident cohort and $26.7 \%$ for the prevalent cohort. These estimates correspond to a doubling of the risk of occurrence of an event throughout the entire treatment period, for incident versus prevalent cohorts (HR 2.19, 95\% CI 1.24-3.87; log-rank $\mathrm{p}=0.006$ ). After adjustment for baseline variables that differed at the $20 \%$ level between the incident and prevalent placebo groups (geographical region, mean right atrial pressure (mRAP) and WHO FC) and aetiology, the HR was 1.96 (95\% CI 1.01-3.83) (online supplementary table S1). 
TABLE 1 Baseline demographic, disease and cardiovascular characteristics of treatment-naïve incident and prevalent cohorts

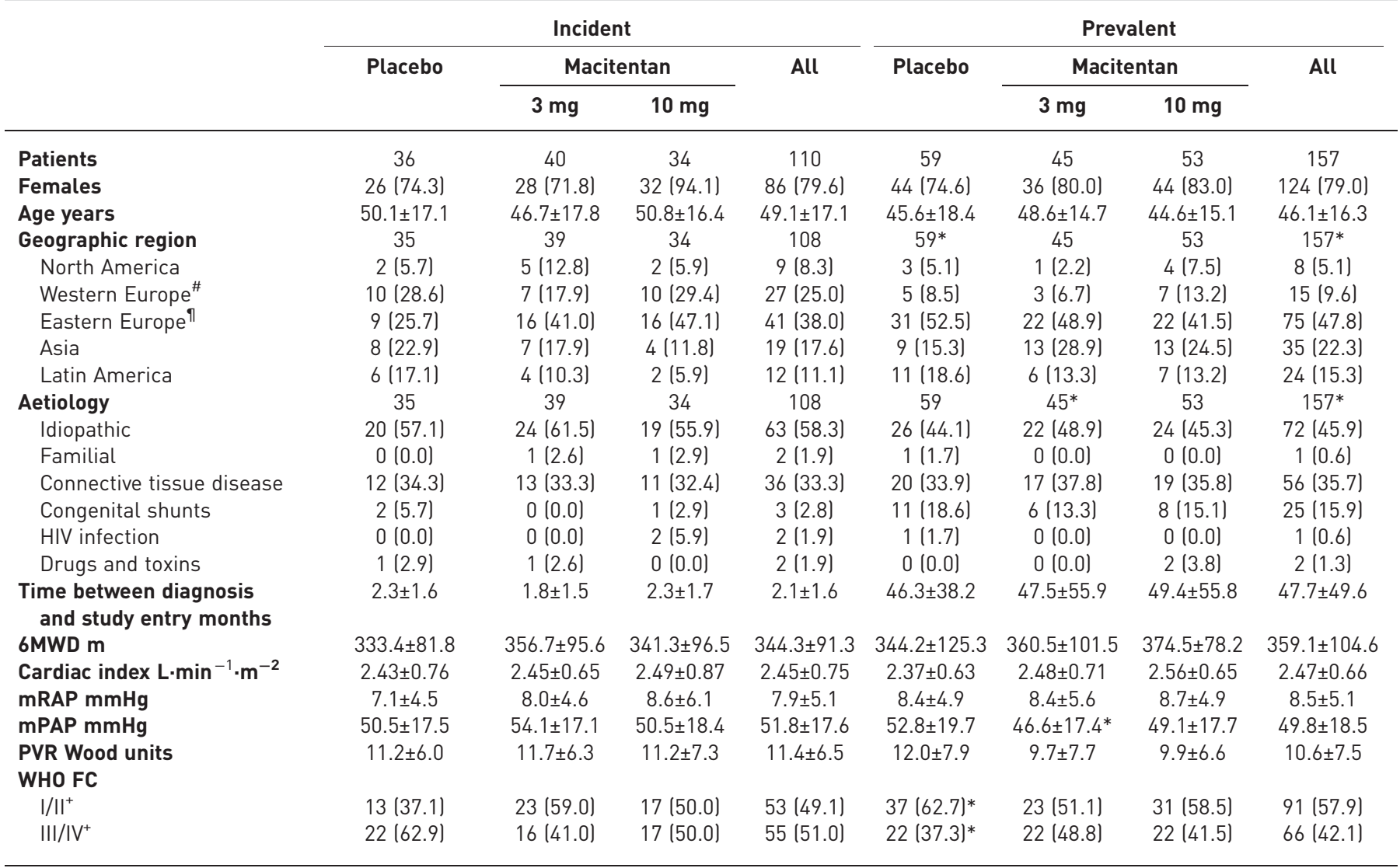

Data are presented as $\mathrm{n}, \mathrm{n}(\%)$ or mean $\pm \mathrm{SD}$, unless otherwise stated. 6MWD: 6-min walk distance; mRAP: mean right atrial pressure; mPAP: mean pulmonary arterial pressure; PVR: pulmonary vascular resistance; WHO: World Health Organization; FC: functional class. ${ }^{\#}$ : includes Israel. $\eta^{\text {: }}$ includes Turkey. ${ }^{+}$: one FC I patient and five FC IV patients were in the overall prevalent cohort; no FC I patients and two FC IV patients were in the overall incident cohort. *: $\mathrm{p}<0.05$ between prevalent and incident patients in the particular treatment group and overall.

The effect of macitentan $10 \mathrm{mg}$ on the composite end-point of morbidity and mortality in the incident and prevalent cohorts versus placebo is shown in figure 1 . Treatment with macitentan $10 \mathrm{mg}$ significantly reduced the risk of a morbidity or mortality event versus placebo by $60 \%$ in incident patients (HR 0.40, 95\% CI $0.20-$ 0.79 ; log-rank $\mathrm{p}=0.007$ ) and by $53 \%$ in prevalent patients (HR $0.47,95 \%$ CI $0.24-0.92$; log-rank $\mathrm{p}=0.023$ ) (figure 2). The test for interaction was nonsignificant $(\mathrm{p}=0.602)$, indicating no heterogeneity of the treatment effect across the incident and prevalent cohorts. The magnitude of the treatment effect observed in the macitentan $3 \mathrm{mg}$ group (versus placebo) was smaller than that of the macitentan $10 \mathrm{mg}$ group (figure S1).

The robustness of these results was assessed by conducting the analysis on all randomised patients irrespective of baseline PAH therapy. Kaplan-Meier estimates of morbidity and mortality at month 12 were $45.4 \%$ versus $23.0 \%$, respectively, for all randomised incident and prevalent patients on placebo (HR 1.72 , 95\% CI 1.16-2.56; log-rank $\mathrm{p}=0.006$ ). There was a significant reduction in the risk of events for incident patients who received macitentan $10 \mathrm{mg}(\mathrm{n}=64)$ versus placebo $(\mathrm{n}=63)$ (57\% risk reduction; HR 0.43 , 95\% CI $0.25-0.73$; log-rank $\mathrm{p}=0.001$ ) and for prevalent patients who received macitentan $10 \mathrm{mg}$ $(\mathrm{n}=177)$ versus placebo $(\mathrm{n}=185)$ (41\% risk reduction; HR 0.59, 95\% CI $0.42-0.84$; log-rank $\mathrm{p}=0.003)$. The magnitude of the treatment effect observed for macitentan $3 \mathrm{mg}$ (versus placebo) was smaller than that of macitentan $10 \mathrm{mg}$.

\section{PAH-related death or hospitalisation end-point}

The total number of PAH-related death or hospitalisation events experienced by patients in the incident and prevalent cohorts is shown by treatment group in table 2. Among the placebo patients, a greater proportion of incident patients experienced a PAH-related death or hospitalisation event compared with prevalent patients; this end-point was mainly driven by hospitalisation for PAH (table 2). Kaplan-Meier estimates of PAH-related death or hospitalisation at month 12 were $47.3 \%$ versus $19.9 \%$ for the incident 
TABLE 2 Morbidity and mortality events and pulmonary arterial hypertension (PAH)-related death and hospitalisation events in treatment-naïve incident and prevalent cohorts

\begin{tabular}{|c|c|c|c|c|c|c|}
\hline & \multicolumn{3}{|c|}{ Incident } & \multicolumn{3}{|c|}{ Prevalent } \\
\hline & \multirow[t]{2}{*}{ Placebo } & \multicolumn{2}{|c|}{ Macitentan } & \multirow[t]{2}{*}{ Placebo } & \multicolumn{2}{|c|}{ Macitentan } \\
\hline & & $3 \mathrm{mg}$ & $10 \mathrm{mg}$ & & $3 \mathrm{mg}$ & $10 \mathrm{mg}$ \\
\hline Patients n & 36 & 40 & 34 & 59 & 45 & 53 \\
\hline $\begin{array}{l}\text { Exposure to study drug weeks median } \\
\text { (interquartile range) }\end{array}$ & $41.2(21.9-97.6)$ & $123.3(50.4-139.0)$ & $122.4(38.4-147.1)$ & $111.0(27.1-146.3)$ & $119.3(46.7-148.3)$ & $122.4(82.3-151.6)$ \\
\hline \multicolumn{7}{|l|}{ Morbidity and mortality } \\
\hline All events & $23(63.9)$ & $16(40.0)$ & 13 (38.2) & $25(42.4)$ & $13(28.9)$ & $13(24.5)$ \\
\hline Worsening of PAH & $18(50.0)$ & $12(30.0)$ & $13(38.2)$ & $17(28.8)$ & $9(20.0)$ & $7(13.2)$ \\
\hline Death & $3(8.3)$ & $4(10.0)$ & 0 & $8(13.6)$ & $4(8.9)$ & $6(11.3)$ \\
\hline Intravenous/subcutaneous prostanoid initiation & $2(5.6)$ & 0 & 0 & 0 & 0 & 0 \\
\hline Lung transplantation & 0 & 0 & 0 & 0 & 0 & 0 \\
\hline Atrial septostomy & 0 & 0 & 0 & 0 & 0 & 0 \\
\hline \multicolumn{7}{|l|}{ PAH-related death or hospitalisation } \\
\hline All events & $18(50.0)$ & $14(35.0)$ & $6(17.6)$ & $17(28.8)$ & $7(15.6)$ & $7(13.2)$ \\
\hline Hospitalisation ${ }^{\#}$ for PAH & $18(50.0)$ & $11(27.5)$ & $6(17.6)$ & $14(23.7)$ & $5(11.1)$ & $4(7.5)$ \\
\hline Death due to PAH & 0 & $3(7.5)$ & 0 & $3(5.1)$ & $2(4.4)$ & $3(5.7)$ \\
\hline All-cause death at EOS & $8(22.2)$ & 7 (17.5) & $4(11.8)$ & 16 (27.1) & $8(17.8)$ & 7 (13.2) \\
\hline
\end{tabular}

Data are presented as $\mathrm{n}(\%)$ unless otherwise stated. EOS: end of study. ${ }^{\#}$ : if a patient died on the day of first hospitalisation or as a result of an adverse event that started on the day of first hospitalisation or on the day of a confirmed worsening of PAH loccurring on the day of first hospitalisation) with a fatal outcome within 4 weeks of the end of treatment, they were counted under hospitalisation for PAH as their first event. 


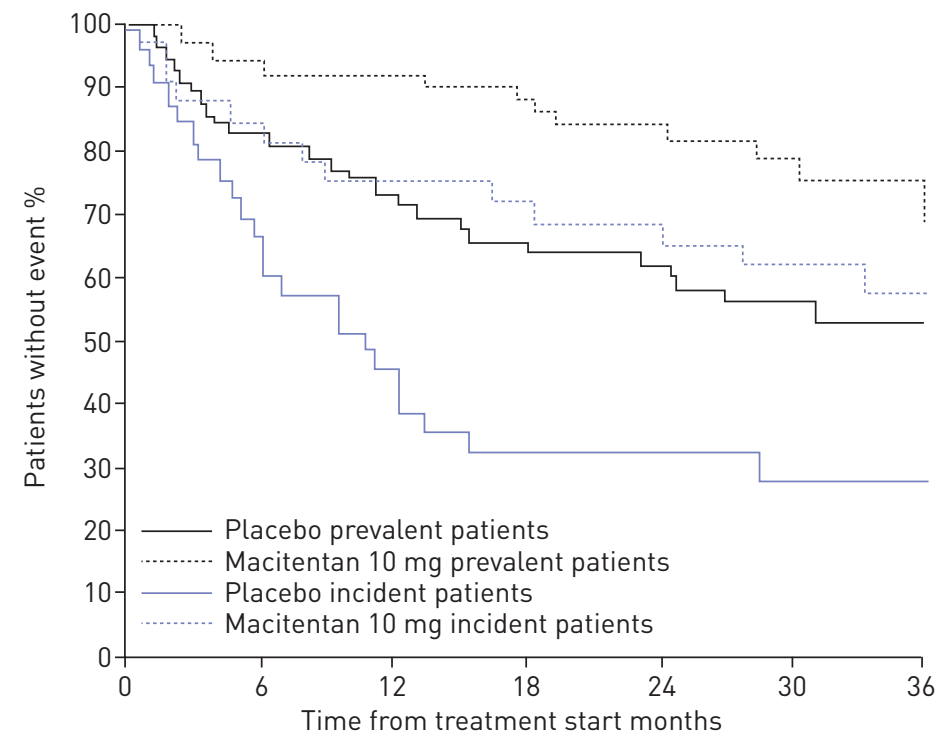

Patients at risk $n$

Placebo incident patients

Macitentan $10 \mathrm{mg}$ incident patients

Placebo prevalent patients

Macitentan $10 \mathrm{mg}$ prevalent patients

$\begin{array}{llll}36 & 20 & 14 & \\ 34 & 27 & 23 & 22 \\ 59 & 46 & 40 & 35 \\ 53 & 47 & 45 & 42\end{array}$

$\begin{array}{cccc}10 & 9 & 4 & 3 \\ 22 & 21 & 16 & 7 \\ 35 & 33 & 20 & 10 \\ 42 & 37 & 22 & 10\end{array}$

FIGURE 1 Effect of macitentan $10 \mathrm{mg}$ on morbidity and mortality in treatment-naïve incident and prevalent cohorts.

Patients/events $\mathrm{n}$

$\begin{array}{ccc}\text { Placebo } & \begin{array}{c}\text { Macitentan } \\ 10 \mathrm{mg}\end{array} & \begin{array}{c}\text { Macitentan } 10 \mathrm{mg} \\ \text { versus placebo } \\ \text { hazard ratio }(95 \% \mathrm{Cl})\end{array}\end{array}$

Time to first confirmed morbidity/mortality event up to EOT

All patients

$95 / 48$

$87 / 26$

$0.447(0.277-0.722)$

Prevalent

$59 / 25$

$36 / 23$

Incident

$53 / 13$

$34 / 13$

$0.467(0.238-0.915)$

$0.396(0.198-0.789)$

Time to death due to PAH or hospitalisation for PAH up to EOT

\begin{tabular}{lccc} 
All patients & $95 / 35$ & $87 / 13$ & $0.310(0.164-0.587)$ \\
& & & \\
Prevalent & $59 / 17$ & $53 / 7$ & $0.382(0.158-0.922)$ \\
Incident & $36 / 18$ & $34 / 6$ & $0.225(0.088-0.573)$ \\
\hline & & \\
Time to death of all causes up to EOS & & \\
All patients & $95 / 24$ & $87 / 11$ & $0.443(0.217-0.904)$ \\
& & \\
Prevalent & $59 / 16$ & $53 / 7$ & $0.423(0.174-1.030)$ \\
Incident & $36 / 8$ & $34 / 4$ & $0.484(0.145-1.608)$
\end{tabular}

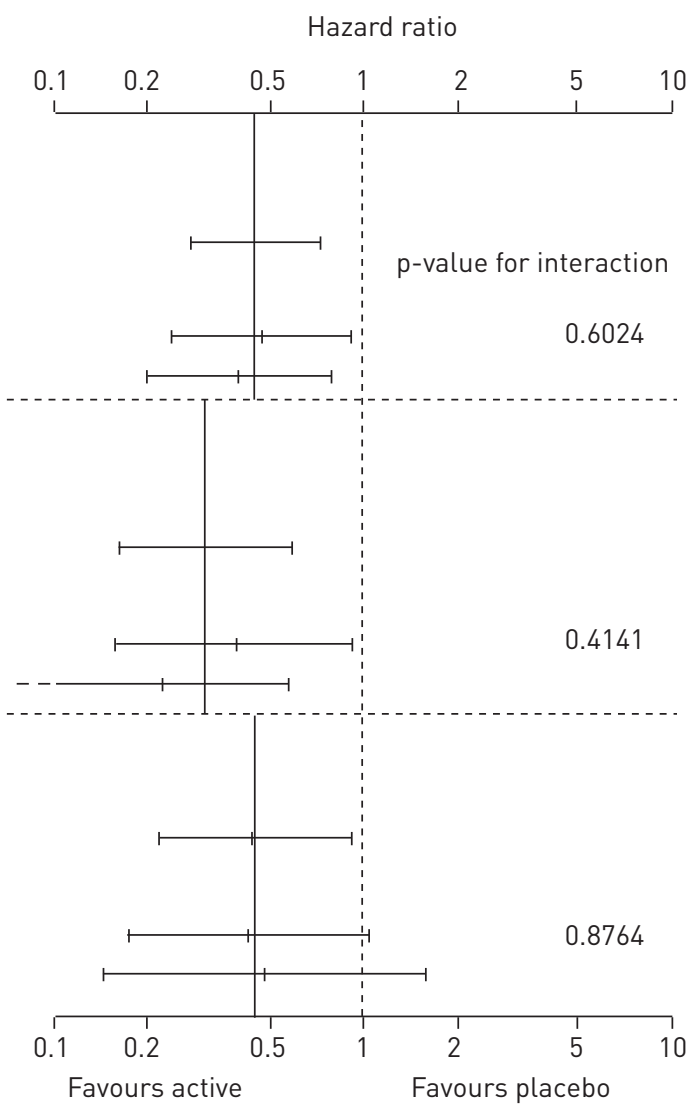

FIGURE 2 Forest plot of risk of time to event end-points in treatment-naïve incident and prevalent cohorts of patients with pulmonary arterial hypertension (PAH) treated with macitentan $10 \mathrm{mg}$ versus placebo. EOT: end of treatment; EOS: end of study. 
and prevalent patients, respectively (figure 3). These estimates correspond to more than twice the risk of occurrence of an event throughout the entire treatment period, for incident versus prevalent patients (HR 2.47, 95\% CI 1.27-4.83; log-rank p=0.006). After adjustment for baseline variables that differed at the $20 \%$ level between incident and prevalent placebo groups (geographical region, mRAP and WHO FC) and aetiology, the HR was 1.98 (95\% CI 0.89-4.43) (table S1).

The effect of macitentan $10 \mathrm{mg}$ on the risk of the composite endpoint of PAH-related death or hospitalisation is shown in figure 3. Treatment with macitentan $10 \mathrm{mg}$ significantly reduced the risk of PAH-related death or hospitalisation by $77 \%$ in incident patients (HR 0.23, 95\% CI 0.09-0.57; log-rank $\mathrm{p}=0.0007$ ) and by $62 \%$ in prevalent patients (HR 0.38, 95\% CI 0.16-0.92; log-rank p=0.026) (figure 2). The test for interaction was nonsignificant $(\mathrm{p}=0.414)$, indicating no heterogeneity of the treatment effect across the incident and prevalent cohorts. The magnitude of the treatment effect observed with macitentan $3 \mathrm{mg}$ (versus placebo) was smaller than that of macitentan $10 \mathrm{mg}$ (figure S1).

\section{All-cause mortality end-point}

The total number of deaths due to any cause in the incident and prevalent cohorts is shown by treatment group in table 2. Time to death from any cause up to EOS for incident and prevalent cohorts is shown in figure 4 .

Although more deaths occurred in the prevalent group than expected, compared with the incident group, there was no statistically significant difference in the risk of all-cause mortality up to EOS between the two cohorts (HR 0.79, 95\% CI 0.34-1.84; log-rank p=0.587). After adjustment for baseline variables that differed at the $20 \%$ level between incident and prevalent placebo groups (geographical region, mRAP and WHO FC) and aetiology, the HR was 0.43 (95\% CI 0.14-1.28) (table S1). Overall, most of the deaths occurred relatively early in the study (table S2a and b).

Baseline characteristics of the patients that died are summarised in table S2a. The functional characteristics (haemodynamic parameters, WHO FC and 6MWD) of the prevalent patients who died were more severe than those of the incident patients who died and more severe than the overall prevalent cohort (tables 1 and S2a and b).

The treatment effect of macitentan $10 \mathrm{mg}$ was examined on the time to all-cause mortality up to EOS in the incident and prevalent cohorts. The HRs for all-cause mortality in patients treated with macitentan $10 \mathrm{mg}$ were 0.48 (95\% CI 0.15-1.61, log-rank p=0.225) and 0.42 (95\% CI 0.17-1.03, log-rank $\mathrm{p}=0.051)$ for the incident and prevalent patients, respectively (figure 2), with no heterogeneity across the incident and

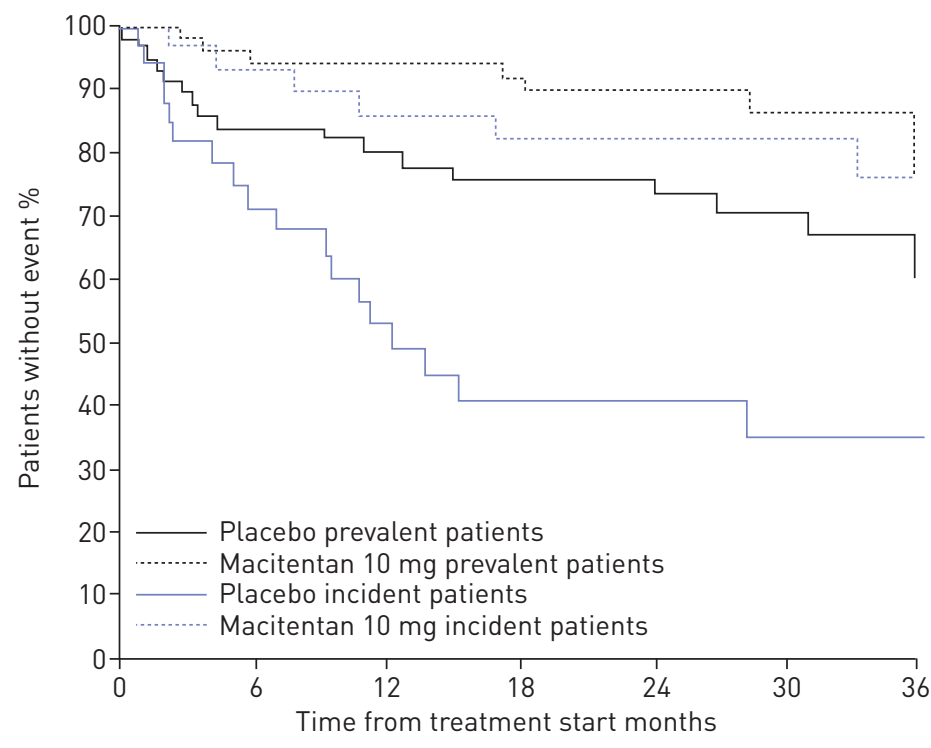

Patients at risk $n$

Placebo incident patients 36

Macitentan $10 \mathrm{mg}$ incident patients $\quad 34$

Placebo prevalent patients $\quad 59$

Macitentan $10 \mathrm{mg}$ prevalent patients

53

$\begin{array}{cccccc}21 & 13 & 10 & 9 & 4 & 3 \\ 26 & 22 & 20 & 20 & 15 & 7 \\ 45 & 39 & 35 & 33 & 20 & 9 \\ 47 & 45 & 42 & 37 & 22 & 10\end{array}$

FIGURE 3 Effect of macitentan $10 \mathrm{mg}$ on pulmonary arterial hypertension-related death or hospitalisation in treatment-naïve incident and prevalent cohorts. 


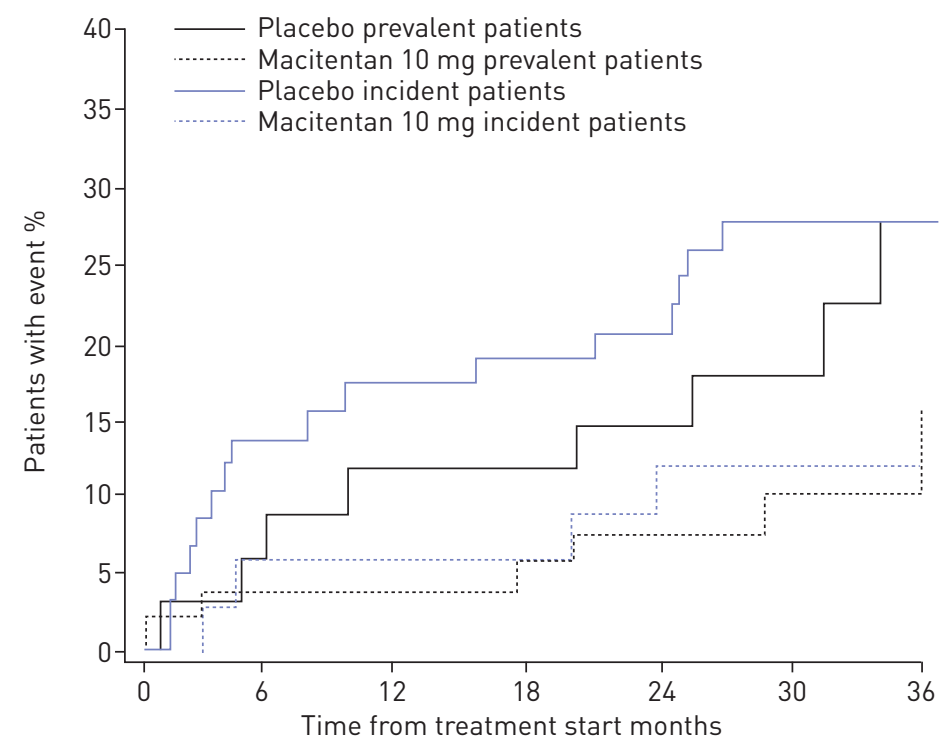

Patients at risk $n$

Placebo incident patients

Macitentan $10 \mathrm{mg}$ incident patients

Placebo prevalent patients

Macitentan $10 \mathrm{mg}$ prevalent patients

36
34
59
53

$\begin{array}{ll}32 & 29 \\ 31 & 31 \\ 50 & 48 \\ 51 & 50\end{array}$

29
31
47
49

$\begin{array}{lll}28 & 19 & 12 \\ 29 & 24 & 11 \\ 46 & 29 & 13 \\ 48 & 30 & 16\end{array}$

FIGURE 4 Effect of macitentan $10 \mathrm{mg}$ on all-cause mortality up to end of study in treatment-naïve incident and prevalent cohorts.

prevalent cohorts (interaction $\mathrm{p}=0.876$ ). The magnitude of the treatment effect for macitentan $3 \mathrm{mg}$ (versus placebo) was smaller than that of macitentan $10 \mathrm{mg}$ (figure S1).

\section{Safety}

Incident patients who received placebo, macitentan $3 \mathrm{mg}$ or macitentan $10 \mathrm{mg}$ were exposed to the study drug for a median (interquartile range) of 41.2 (21.9-97.6), 123.3 (50.4-139.0) and 122.4 (38.4-147.1) weeks, respectively. Prevalent patients were exposed to placebo, macitentan $3 \mathrm{mg}$ or macitentan $10 \mathrm{mg}$ for a median of 111.0 (27.1-146.3), 119.3 (46.7-148.3) and 122.4 (82.3-151.6) weeks, respectively (table 3). Adverse events associated with ERAs, such as nasopharyngitis, respiratory tract infections, oedema and headache, were more common in patients receiving macitentan than placebo, irrespective of whether they were incident or prevalent (table 3). There was a greater incidence of syncope and right ventricular failure in placebo incident patients than in placebo prevalent patients. The incidence of clinical laboratory abnormalities was low in each cohort across treatment groups.

\section{Discussion}

In this post hoc analysis of treatment-naïve patients in the SERAPHIN study, a greater proportion of incident patients experienced disease progression events compared with prevalent patients, yet there was no difference in the risk of all-cause death between the cohorts. The differences in disease progression were observed despite there being no clinically significant differences in baseline haemodynamics between the incident and prevalent cohorts. During the study, macitentan $10 \mathrm{mg}$ improved long-term outcomes in both incident and prevalent patients, as evidenced by a reduction in the risk of disease progression events, regardless of the time from diagnosis.

Data from national PAH registries in France and in the USA highlight that newly diagnosed PAH patients have a worse prognosis than prevalent patients $[2,4]$. This analysis of incident and prevalent cohorts from SERAPHIN, which is the first time, to our knowledge, that a comparison of outcomes in these subgroups has been evaluated in a randomised controlled setting, provides similar observations for disease progression based on two composite end-points: time to first morbidity or mortality event and time to first PAH-related death or hospitalisation. These end-points were driven by PAH worsening and hospitalisation for PAH, respectively; the number of deaths contributing to each of these end-points was low, as patients usually experience progression of their disease before death.

The analysis in this global study showed no difference between the two cohorts in terms of all-cause mortality up to EOS. These findings were unexpected, since registry data have shown poorer survival 
TABLE 3 Exposure to study drug, most frequent adverse events and laboratory abnormalities in treatment-naïve incident and prevalent cohorts

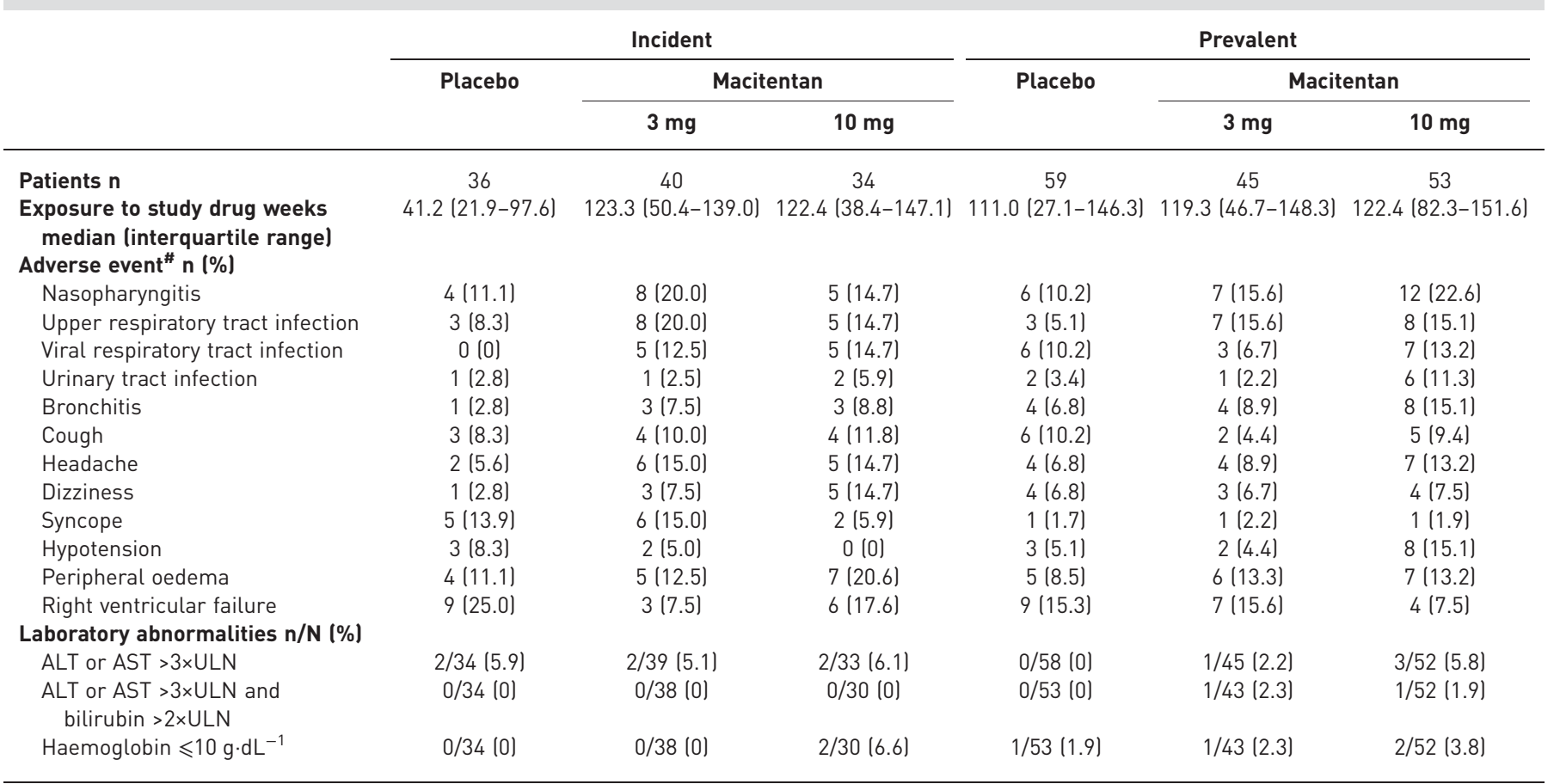

ALT: alanine aminotransferase; AST: aspartate aminotransferase; ULN: upper limit of normal. ${ }^{\#}$ : occurring in $>10 \%$ of at least one treatment arm in either cohort.

among incident patients compared with prevalent patients $[2,4,5]$. Nevertheless, prevalent patients still experience disease worsening and deteriorate over time. The prevalent cohort had a mean time from diagnosis to enrolment of $\sim 4$ years, indicating that some patients may have been approaching the end stage of their disease. The Kaplan-Meier plot shows that most of these deaths occurred in the first 6 months. Notably, the majority of the deaths in the prevalent cohort occurred in countries where access to PAH treatment may be limited. The baseline characteristics of the prevalent patients who died in this study, including cardiac index, mRAP, FC and 6MWD, which are prognostic for survival [17], suggest that their disease was more advanced compared with the overall population. The observed outcomes and geographical differences of the incident and prevalent cohorts indicate a discrepancy between incident and prevalent outcomes in countries that have access to PAH therapies and countries where access may be limited. Participation in a clinical trial may be the only treatment alternative for patients in the latter. These findings highlight important differences in the natural history of these disparate populations that should be considered in the design of future clinical trials.

The observed safety profile of macitentan $10 \mathrm{mg}$ in the incident and prevalent cohorts was consistent with that of the overall study population [13]. Adverse events in the overall SERAPHIN population that were more frequently associated with macitentan than placebo were headache, nasopharyngitis and anaemia. In the current analysis of incident and prevalent cohorts, respiratory tract infections were slightly higher with active treatment versus placebo. In the treatment-naïve incident patients, peripheral oedema was reported more frequently with macitentan $10 \mathrm{mg}$ than placebo. These data should be interpreted within the context of the substantially longer exposure to the active drug compared with placebo (122 versus 41 weeks).

These analyses have several potential limitations. They are exploratory, and the sample size in the subgroups should be considered. In addition, as patients were not stratified by time from diagnosis or background PAH therapy (robustness analysis), there was potential for imbalance between treatment groups. The difference in outcome between the incident and prevalent placebo cohorts may be accentuated by the slight imbalance in baseline WHO FC. Other baseline characteristics not assessed in the study could account for differences in outcome between the incident and prevalent patients. In addition, the potential for a survivor bias and its impact on the results should be considered. There is probably an over-representation of survivors in the prevalent group, as this group is composed of patients who survived for $\geqslant 6$ months after their initial diagnosis. In contrast, the incident group is likely to be composed of a mixture of patients who rapidly 
decline and those who have better outcomes. The latter patients in the incident group would, if sampled at a later stage, be included in the prevalent group. A similar survivor bias has been observed in other diseases, including some cancers $[7,18,19]$. Geographical differences in treatment availability could also influence outcomes. Overall, there were few deaths contributing to the disease progression composite end-points; the results should be interpreted with caution.

These data indicate a higher risk for $\mathrm{PAH}$ progression in incident patients compared with prevalent patients, although the risk of death between groups was similar. Nevertheless, these findings highlight the importance of initiating treatment in patients as soon as PAH is diagnosed. Furthermore, the analysis provides evidence to support the use of macitentan as an effective first-line therapy for delaying disease progression in both newly diagnosed and prevalent patients with PAH.

\section{References}

1 Galiè N, Manes A, Negro L, et al. A meta-analysis of randomized controlled trials in pulmonary arterial hypertension. Eur Heart J 2009; 30: 394-403.

2 Humbert M, Sitbon O, Yaici A, et al. Survival in incident and prevalent cohorts of patients with pulmonary arterial hypertension. Eur Respir J 2010; 36: 549-555.

3 Humbert M, Sitbon O, Chaouat A, et al. Survival in patients with idiopathic, familial, and anorexigen-associated pulmonary arterial hypertension in the modern management era. Circulation 2010; 122: 156-163.

4 D'Alonzo GE, Barst RJ, Ayres SM, et al. Survival in patients with primary pulmonary hypertension. Results from a national prospective registry. Ann Intern Med 1991; 115: 343-349.

5 Miller DP, Farber HW, Poms AW, et al. Five-year outcomes of the Registry To Evaluate Early And Long-Term Pulmonary Arterial Hypertension (PAH) Disease Management (REVEAL). Am J Respir Crit Care Med 2014; 183: A4741.

6 McGoon MD, Benza RL, Escribano-Subias P, et al. Pulmonary arterial hypertension. J Am Coll Cardiol 2013; 62: D51-D59.

7 Miller DP, Gomberg-Maitland M, Humbert M. Survivor bias and risk assessment. Eur Respir J 2012; 40: 530-532.

8 Gatfield J, Mueller Grandjean C, Sasse T, et al. Slow receptor dissociation kinetics differentiate macitentan from other endothelin receptor antagonists in pulmonary arterial smooth muscle cells. PLoS One 2012; 7: e47662.

9 Gatfield J, Mueller Grandjean C, Bur D, et al. Distinct ETA receptor binding mode of macitentan as determined by site directed mutagenesis. PLoS One 2014; 9: e107809.

10 Iglarz M, Binkert C, Morrison K, et al. Pharmacology of Macitentan, an Orally Active Tissue-Targeting Dual Endothelin Receptor Antagonist. J Pharmacol Exp Ther 2008; 327: 736-745.

11 Iglarz $\mathrm{M}$, Bossu $\mathrm{A}$, Wanner $\mathrm{D}$, et al. Comparison of pharmacological activity of macitentan and bosentan in preclinical models of systemic and pulmonary hypertension. Life Sci 2014; 118: 333-339.

12 Actelion Pharmaceuticals US, Inc. Opsumit (macitentan) prescribing information. https://opsumit.com/sites/ opsumit/files/OPSUMIT-Full-Prescribing-Information.pdf Date last accessed: March, 2015. Date last updated: April, 2015.

13 Pulido T, Adzerikho I, Channick RN, et al. Macitentan and morbidity and mortality in pulmonary arterial hypertension. N Engl J Med 2013; 369: 809-818.

14 Jansa P, Jarkovsky J, Al-Hiti H, et al. Epidemiology and long-term survival of pulmonary arterial hypertension in the Czech Republic: a retrospective analysis of a nationwide registry. BMC Pulm Med 2014; 14: 45.

15 Escribano-Subias P, Blanco I, López-Meseguer M, et al. Survival in pulmonary hypertension in Spain: insights from the Spanish registry. Eur Respir J 2012; 40: 596-603.

16 Thenappan T, Shah SJ, Rich S, et al. A USA-based registry for pulmonary arterial hypertension: 1982-2006. Eur Respir J 2007; 30: 1103-1110.

17 McLaughlin VV, Gaine SP, Howard LS, et al. Treatment goals of pulmonary hypertension. J Am Coll Cardiol 2013; 62: D73-D81.

18 Gallagher DJ, Gaudet MM, Pal P, et al. Germline BRCA mutations denote a clinicopathologic subset of prostate cancer. Clin Cancer Res 2010; 16: 2115-2121.

19 Kelsey KT, Hankinson SE, Colditz GA, et al. Glutathione S-transferase class mu deletion polymorphism and breast cancer: results from prevalent versus incident cases. Cancer Epidemiol Biomarkers Prev 1997; 6: 511-515. 